\title{
The Current Clinically Relevant Findings on COVID-19 Pandemic
}

\author{
Joseph V. Pergolizzi Jr. ${ }^{1}$, Peter Magnusson (iD ${ }^{2,3}$, Jo Ann LeQuang (DiD ${ }^{1}$, Frank Breve ${ }^{1}$, Antonella Paladini \\ (iD) ${ }^{4}$, Martina Rekatsina ${ }^{5}{ }^{5}$, Cheng Teng Yeam (iD) ${ }^{6}$, Farnad Imani (iD) ${ }^{7}$, Giorgia Saltelli ${ }^{8}$, Robert Taylor Jr. \\ (D) ${ }^{1}$, Charles Wollmuth ${ }^{1}$ and Giustino Varrassi (iD) ${ }^{9, *}$ \\ ${ }^{1}$ NEMA Research, Inc., Naples. Florida, USA \\ ${ }^{2}$ Centre for Research and Development, Uppsala University/Region Gävleborg, Gävle, Sweden \\ ${ }^{3}$ Cardiology Research Unit, Department of Medicine, Solna, Karolinska Institutet, Stockholm, Sweden \\ ${ }^{4}$ Department MESVA, University of L'Aquila, 67100 L'Aquila, Italy \\ ${ }^{5}$ Anaesthetic Fellow, Moorfields Eye Hospital, London, UK \\ ${ }^{6}$ Duke-NUS Medical School, 8 College Road, Singapore 169857, Singapore \\ ${ }^{7}$ Pain Research Center, Iran University of Medical Sciences, Tehran, Iran \\ ${ }^{8}$ Sant'Andrea Hospital, La Sapienza University, COVID Section, Roma, Italy \\ ${ }^{9}$ Paolo Procacci Foundation, Via Tacito 7, 00193, Rome, Italy \\ "Corresponding author: Paolo Procacci Foundation, Via Tacito 7, 00193, Rome, Italy. Email: giuvarr@gmail.com
}

Received 2020 April 15; Accepted 2020 April 16.

\begin{abstract}
The emergence of a novel coronavirus and coronavirus disease 2019 (COVID-19) represents a challenge to global healthcare. In the past 20 years, this is the third coronavirus that jumped the species barrier and infected humans. It is highly contagious but associated with low pathogenicity. First identified in Wuhan, China, a city of over 11 million, the disease has since spread to every continent except Antarctica. About $15 \%$ to $20 \%$ of all cases may be called severe, and it is believed many cases are asymptomatic. The average age of a person with COVID has been reported as 49 years. Worse outcomes are associated with geriatric populations and those with underlying diseases such as cardiovascular, respiratory disorders, and/or diabetes. The coronavirus, like other coronaviruses, is highly contagious and has a latency period of about 14 days. Most patients present with fever and a dry cough, but fever may be absent. Differential diagnosis can be challenging since influenza may present with similar symptoms. Chest radiography or computed tomography may be used to find evidence of secondary pneumonia. Nosocomial infection is of concern, and it has been reported that $3.8 \%$ of all cases with COVID-19 in that country involve healthcare workers in China. Most patients have mild disease, and supportive care suffices. A variety of repurposed and investigational drugs are being evaluated. There are currently no antiviral therapies or vaccines, even if many therapies are proposed. Hand hygiene, social distancing, and scientifically sound information are the best strategies at the moment to combat this epidemic.
\end{abstract}

Keywords: Infection, MERS, Corticosteroids, Anti-inflammatory, NSAIDs, COVID-19, Coronavirus, SARS

\section{Context}

In February 2018, the World Health Organization (WHO) published its Blueprint List of priority diseases that includes Severe Acute Respiratory Syndrome (SARS), Middle Eastern Respiratory Syndrome (MERS), and a to-beidentified disease listed in its report as Disease X. Disease $\mathrm{X}$ was defined as a new disease with epidemic or pandemic potential caused by an unknown pathogen (1). The emergence of the novel coronavirus in 2019, now known as SARSCoV-2 or COVID-19, may be the Disease X. In our era of globalization, mass travel and relatively porous borders cause a lot of concerns. This virus is the seventh identified form of coronavirus that can cause respiratory disease in humans (2). Its emergence should be of no surprise such that this is the third example of a zoonotic coronavirus that jumped the species barrier to infect numerous humans across the globe in the past 20 years (3), the others include SARS in 2003 and MERS in 2015 (4-6).

For most adults and children with good health, the virus manifests with mild flu-like symptoms and it appears many infected individuals are asymptomatic or have such mild symptoms that they do not seek medical care. For vulnerable populations and those with underlying health problems, the illness may be severe, resulting in multiple organ failure and death (7). Our current understanding is that the routine latency period for COVID-19 takes three to seven days with a maximum of 14 days $(8,9)$. It appears that unlike SARS, COVID-19 can be contagious even during the latency period (8). At first, it was thought that pediatric patients did not get COVID-19 or that their immune systems 
were particularly robust at holding back the pathogen, but a study of 391 individuals and their close contacts $(n=1,286)$ found that children under the age of 10 were just as likely to contract the COVID-19 as people of other age groups and that about $7 \%$ of these children exposed to the virus became infected (10). Until April, no deaths have been reported in any patient younger than nine years (11). Unfortunately, a few studies reported the deaths of newborns, infants, and toddlers (12-14). It is not yet clear how efficient the children are at transmitting of SARS-CoV-2 (10).

The aim of this study was to summarize the current understanding of the clinically relevant findings on COVID-19.

\section{COVID-19 in the Context of Other Coronaviruses}

Human coronaviruses are common, enveloped RNA viruses that cause up to a third of all common colds in adults (15). Low pathogenic types of the virus mainly affect the upper respiratory system and produce mild symptoms. In contrast, highly pathogenic types, such as SARS and MERS, can cause lower-respiratory tract infections and lead to secondary life-threatening bacterial pneumonia (16). In cases of highly pathogenic SARS or MERS infection, the virus replicates rapidly with massive infiltration of inflammatory cells and an escalated pro-inflammatory cytokine response, known as cytokine storm, which is an aberrant immunological response $(16,17)$. A suppressed immune system or inadequate immune response may facilitate viral replication and attendant tissue destruction. On the other hand, an over activated inflammatory and immunological defense may set the stage of immunopathologies (18). The SARS-CoV-2 is a beta-coronavirus of low pathogenicity; however, it has a phylogenetic similarity to the SARS (19). The SARS-CoV-2 is a non-segmented, positivesense, single-stranded RNA virus with a nucleocapsid composed of genomic RNA and a phosphorylated nucleocapsid protein. The virion is located in the phospholipid bilayers and is covered by spike proteins. The virus family takes its name from the "crowns" that appear on the virus (Figure 1) (3). These surface glycoprotein spikes are thought to be crucial for the virus to bind to the host, possibly restricting the range of potential hosts (5). Genomic sequencing of SARS-Cov2 was performed on bronchoalveolar lavage fluid, and cultured isolates from nine confirmed COVID-19 inpatients (20). The ten genome sequences that resulted had over $99.98 \%$ sequence identity and $88 \%$ similarity to coronavirus sequences obtained from two bats with SARS but were less similar to MERS ( 50\%) (20). The SARS-Cov2 is in the beta-coronavirus genus in the subgenus Sarbecovirus with sufficient genetic originality to be considered a novel virus (20). The genetic sequences of the novel coronavirus have been published on the Global Initiative on Sharing All Influenza Data (GISAID) at gisaid.org (3).

The COVID-19 virus is enveloped within round or oval particles having a diameter of about 60 to $140 \mathrm{~nm}$. As coronaviruses generally are ubiquitous pathogens, many people already have natural corresponding antibodies. For example, it has been estimated that $30 \%$ to $60 \%$ of the mainland Chinese population has some forms of anti-COVID-19 antibodies (18). It appears that, like SARS, SARS-Cov2 receptor is the angiotensin-converting enzyme 2 (ACE2) receptor that is highly expressed on the epithelium of the airways, which mediates cellular entry $(8,20)$. The SARS-Cov2 replicates more rapidly in human airway epithelial cells than in standard-tissue cultures (19). COVID-19 seems to be more readily transmissible than SARS or MERS (11).

The SARS-Cov2 was first identified in the city of Wuhan, China in December 2019 (21). Wuhan is a large, landlocked city of about 11 million inhabitants and is the capital of Hubei Province. Compared to SARS and MERS, COVID-19 is associated with milder illness and lower fatality rates; the case fatality rate for SARS is $9.5 \%$, and MERS is $34.4 \%$ (6).

The SARS originated from China, and it may be that lessons learned from that epidemic played an important role in Chinese response to COVID-19. China did not report SARS to the WHO until there were 300 confirmed cases, and five deaths had occurred. In contrast, China reported COVID-19 to WHO with just 27 confirmed cases and before any fatalities occurred (11). Thus, the lag time from pathogen identification to WHO reporting went from two months with SARS to a week with COVID-19 (11). China also acted quickly to impose travel restrictions as the outbreak of COVID-19 coincided with Christian New Year, the most important holiday of the year and associated with parties, large gatherings, and travel (11). Even outside Wuhan, tourist attractions such as the Forbidden City in Beijing and Disneyland parks in Shanghai and Hong Kong were closed quickly (22).

\section{The COVID-19 Disease Pyramid}

The extent of subclinical COVID-19 presentation remains unknown, so it is not yet possible to construct a "disease pyramid" to evaluate the relationship of asymptomatic to mild to moderate and severe cases (6). Deaths seem to occur in geriatric or other vulnerable patients and/or immunocompromised patients and relate to acute respiratory distress syndrome (2). It has been estimated that about $15 \%$ to $20 \%$ of cases with COVID-19 may be categorized as severe, but that may be an inaccurate assessment because a number of asymptomatic cases which do not seek medical attention are unknown and may even compose the majority of all cases (2). 


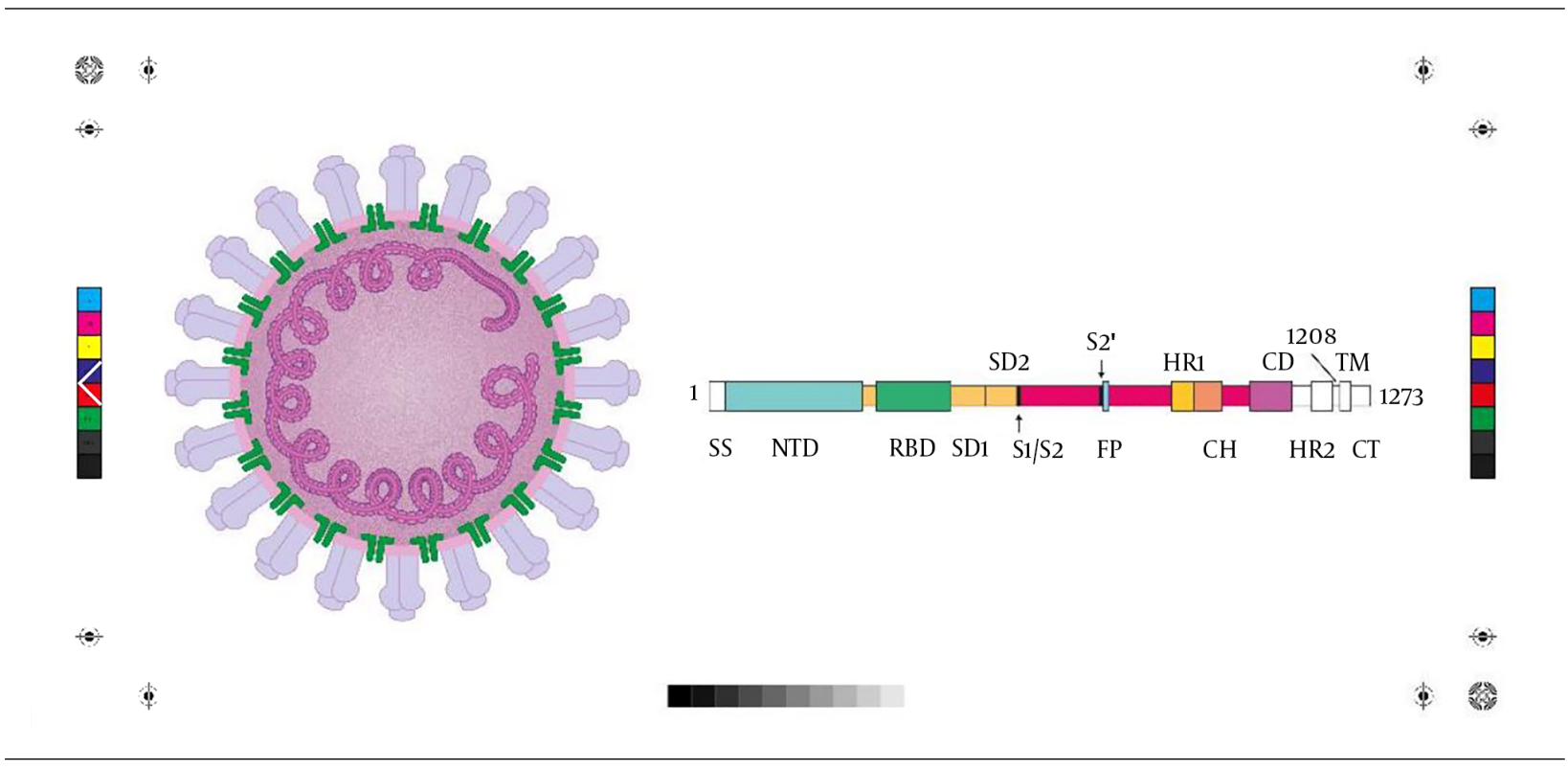

Figure 1. New Coronavirus (SARS-CoV-2 or COVID-19), and its amino acids sequence

Early epidemiological data from China have found that the average age at which a person contracts COVID-19 is 49 years (21), and most cases occur in people between the ages of 30 and 79 years (11). Only about $1 \%$ of cases occurred in patients under the age of nine (11). Of all confirmed cases, $81 \%$ could be described as "mild" (11). In one of the first epidemiological reports from China, of 41 hospitalized patients in the city of Wuhan, 30/41 were men and $32 \%$ had an underlying disease (17). A later study $(n=1,099)$ on laboratory-confirmed cases with COVID-19 showed that of 552 hospitals throughout China, 58.1\% of patients were men, and the median age of all patients was 47 years (23). The composite endpoint, met by $6.1 \%$ of patients $(n=67)$, was the admission to intensive care unit (ICU), use of mechanical ventilation, or death (23).

Based on a study on 1,099 confirmed cases with COVID19 , the median incubation period of the disease is 4 days, with an interquartile range of 2 to 7 days (23). The main symptoms of COVID-19 are fever, dry cough, and dyspnea. It should be noted that fever does not necessarily need to be present. Nasal congestion and rhinorrhea are not present in all patients but have been reported. Patients with severe disease may exhibit weakened breath sounds (9). Differential diagnosis can be challenging because many viral respiratory diseases produce similar symptoms (24). This is further complicated by the fact that bacterial pneumonia, which may be secondary to COVID-19, may be caused by other factors (24).

\section{A Short Timeline of COVID-19}

For many years, the coronavirus was regarded as a ubiquitous but clinically inconsequential pathogen associated with the annoying but hardly dangerous common cold (5). The versatile coronavirus has infected a range of animal species and zoonotic transmission, although rare in general, has played a major role in the emergence of novel human coronaviruses and ensuing epidemics (25). The first patient with COVID-19 was one in a cluster of patients suffering from "pneumonia of unknown origin" in early December 2019 (26). Patient zero was likely to have been infected at the Hua'nan Seafood Wholesale Market in Wuhan (4). Although nominally a wholesale market for seafood, the market actually carried a wide range of living and dead wild animals (26). Bats, which are sold for human consumption in Wuhan, have been implicated as a possible host for the COVID-19 virus but it is also possible that some other intermediate animals were involved (17). Further evidence for bats as the possible animal host was obtained when a live SARS-related coronavirus was isolated from a fecal sample from a bat with 99.9\% whole genome sequence identity to SARS was identified in horseshoe bats (27). Contact tracing revealed that many of those with this mysterious pneumonia had recently gone to the Hua'nan Seafood Wholesale Market. By the time, the first fatality was reported on January 1, 2020, the market was closed for decontamination and other wildlife markets in the area were closed (26).

The pathogen was isolated on January 7, 2020, which 
was initially named "2019 novel coronavirus" or 2019-nCoV and the disease was named on January 12, 2020, by WHO as the coronavirus disease of 2019 or COVID-19 (9). The isolation of the virus allowed Chinese scientists to develop a rapid testing method for point-of-care (21). The first fatality in a person confirmed to have the SARS-CoV2 occurred on January 11, 2020. On January 12, 2019, a whole genome sequence was shared with WHO (21), and by January 13, 2020, the disease had spread to Thailand with other cases soon reported in Japan, Korea, Vietnam, Singapore, Taiwan, Hong Kong, Macau, and the United States (21). On January 30,2020 , WHO declared it a public health emergency of international concern (26).

Wenliang, a physician in China, was arrested when he raised questions about COVID-19 on social media. He contracted the virus and died on February 7, 2020 (28). By February 11, China had launched its so-called "wartime control measures" to put cities on lockdown, essentially restricting the movements of an estimated 760 million citizens (28). Flights and trains in and out of Wuhan were canceled, schools were closed, and even private cars were restricted (2). Because of Christian New Year festivities, which occur over many days, it is estimated that about 5 million people who normally resided in Wuhan were away from home when the quarantine was announced (2).

The disease spread with 75,748 confirmed cases with COVID-19 was reported in 28 countries by February 20 , 2020 , but the vast majority of those cases $(\sim 99 \%)$ were in mainland China (26). Fourteen cases were reported in the United States on February 21, 2020, and by March 5, 2020, the United States had 150 confirmed cases of COVID-19 and 11 deaths (29). February 26, 2020, marked the first date where there were more confirmed new cases with COVID19 outside of mainland China than within (30). The disease was spread to every continent except Antarctica. By March 5,2020 , there were 93,090 confirmed cases with COVID-19 around the world with 80,422 in China; 2,502 in Italy; 212 in France; 24 in Sweden; 16 in Iceland; and 8 in Belgium, highlighting the rapid spread of the disease (31). At the moment, the world number of diagnosed cases is over 1.5 Million.

\section{Transmissibility}

Coronaviruses, in general, tend to be highly contagious; in one case during the SARS epidemic, one person traveling from Hong Kong to Toronto could be traced to 128 cases with SARS in a local hospital; the same occurred with MERS where one person from Saudi Arabia who visited South Korea was associated with 186 cases with MERS (6). With SARS and MERS, most infections were the result of nosocomial transmission within the hospital setting, and this suggests that there is likely high risk of nosocomial transmission of COVID-19 (6). COVID-19 disease transmission is thought to occur by droplets in liquid or aerosol form from one infected person to another person (4). Evidence is emerging that asymptomatic individuals infected with COVID-19 may be capable of transmitting the disease (32). There is also some evidence that patients may continue to shed the virus after recovery (32). Additionally, it may be that "super-spreader" events may occur in which one infected individual may be able to transmit the virus to a disproportionately large group of people (6). While the original epidemic centered on Wuhan and the seafood market, today at least $72 \%$ of people newly infected with COVID-19 have no ties to Wuhan province or its residents (23).

The basic reproductive number (R0) is defined as the expected number of cases that are directly generated by one case among a population of susceptible individuals. The Ro for COVID-19 is approximately 1.4 to $2.5(2,3)$. From a study conducted in China from December 31, 2019, to January 28,2020 , the Ro was 2.68 (95\% confidence interval, 2.47 to 2.86 ) and the epidemic population doubled every 6.4 days (95\% confidence interval, 5.8 to 7.2 ) (33).

Since COVID-19 can be efficiently transmitted from human to human, an epidemic is made possible; globalization and increased travel allows for the pandemic. The fact that COVID-19 is overall associated with milder symptoms than other viruses may mean it will spread faster (6). For example, during the $\mathrm{H} 1 \mathrm{~N} 1$ (swine flu) epidemic, the virus caused relatively minor upper respiratory symptoms but spread extensively. By contrast, the H7N9 (avian flu) virus was associated with a far more virulent lower-respiratory tract infection and had a $40 \%$ fatality rate but was not as readily transmissible (6). The lower fatality rate exhibited by COVID-19 allows for the preservation of infected hosts, which can increase the potential for continued transmission.

People who may have been exposed to COVID-19 should be observed for 14 days, during which time they should have minimal contact with other people. The 14-day window is counted forward from the last day of possible exposure (9). Although patients with confirmed cases of COVID19 may be encouraged to wear $\mathrm{N} 95$ protective masks, there is little evidence that masks worn by unexposed people serve any protective purpose (9). When a suspected person with or confirmed case with COVID-19 requires hospitalization, the patient should as much as possible travel in a private vehicle, wear a mask, and keep distant from other individuals; the hospital should be alerted of the arrival of a possible or confirmed patient with COVID-19 before the patient is brought to the facility (9).

If a patient is isolated at home, he or she should stay in 
a well-ventilated single room cleaned multiple times a day with a solution of $500 \mathrm{mg} / \mathrm{L}$ chlorine disinfectant (9). Towels, silverware, dishes, and other personal items should be single-use only or thoroughly washed after each use (9). Thorough and frequent hand-washing with soap and water is recommended for both patients and households. If a person isolated at home does not improve quickly or if symptoms worsen, medical care may be warranted (9).

\section{Clinical Course and Diagnosis}

In a study of 80 patients with diagnosed COVID-19 in China from January to February 2020, the most common clinical presentations included elevated body temperature (76\%) and coughing (73\%). The clinical trajectory for adults and children in otherwise good health often involves mild symptoms and it is thought that many are unaware they have the virus. In vulnerable patients, the elderly, or those with underlying diseases, pneumonia, or other severe and potentially life-threatening respiratory symptoms may develop (34). In a study of 1,099 confirmed cases with COVID19 in Chinese hospitals, $43.8 \%$ of patients had fever upon admission to the hospital and $88.7 \%$ developed a fever during their hospital stay; $67.8 \%$ had a cough, and 3.8\% had diarrhea (23).

Diagnosis is based on case presentation (fever and symptoms of respiratory illness) combined with an epidemiological link to the virus in the past 14 days (34). As the disease spreads, this epidemiologic link becomes increasingly tenuous. It should also be noted that not all patients develop a fever. The standard test is a nucleic acid test used with respiratory or serum samples, which can confirm or refute COVID-19 infection in about four to six hours $(7,9)$.

Chest radiography or chest computed tomography (CT) can be used to find evidence of pneumonia, which typically presents in the form of bilateral, ground-glass opacity in more than one lobe and with sub-segmental areas of consolidation (35). In fact, chest CT or radiography has been recommended to confirm COVID-19 (9). A study of 80 patients with COVID-19 diagnosed between January and February 2020, found that on chest CT, 91\% had evidence of ground-glass opacity, $63 \%$ had consolidation, and $59 \%$ showed interlobular septal thickening. Most lesions were multiple with an average of $12 \pm 6$ segments (35). In this study, the average pulmonary inflammation index (PII) was $34 \% \pm 20 \%$ and could be significantly correlated with lymphocyte count, monocyte count, C-reactive protein levels, procalcitonin, days from onset of illness, and body temperature $(\mathrm{P}<0.05)(35)$. In a study of 1,099 patients with COVID-19, 56.4\% had evidence of ground-glass opacity on chest CT, which was considered the most common radiologic finding in the confirmed patients (23). It must be noted in this context that patients without severe disease may have normal chest CT or radiography. In fact, $2.9 \%$ of patients with severe COVID-19 had normal chest images and $17.9 \%$ of those with milder forms of COVID-19 had normal images (23).

In a study of chest CTs in patients with COVID-19 $(n=21)$, 3/21 patients had normal chest CTs. Of the patients with abnormal chest CTs, $86 \%$ had the disease, affecting more than one lobe and $38 \%$ had the disease in all five lobes (36). The mean total lung severity score was 9.9 (range 0 to 19) and the patient with the highest score in this study required hospitalization in the ICU (36). A follow-up CT scan was carried out in these patients at a mean of 2.5 days later (range 1 to 4 days) and no patient showed severe progression and none showed interval improvement; the majority (63\%) showed signs of mild progression and one of the patients who had a normal CT scan for the first time exhibited one peripheral ground-glass lesion on a CT scan three days later (36).

\section{Vulnerable Populations}

Most people who are infected with COVID-19 recover. Severe morbidity and mortality appear to occur in the vulnerable populations who contract COVID-19.

\subsection{Neonates}

In early February 2020, China reported the infection in a neonate 30 hours old (7). This caused China to launch a special initiative in its neonatal intensive care units with specific diagnostic and discharge criteria (7). Neonates with risk factors for COVID-19 such as family history or exposure should be isolated in a single room for 14 days (7). If an infant had previously shared a room with other babies, the others in the group should also be isolated until COVID-19 can be definitively ruled out (7). Any sort of disease outbreak in pediatric wards will be associated with extreme psychological distress in parents. As much as possible, social workers or trained psychological staff should be available to counsel parents and family members in case of any sort of viral outbreak in the NICU or in pediatric care centers (7).

\subsection{Pediatric Patients}

The COVID-19 does not seem to be prevalent in pediatric patients but at least 20 pediatric cases have been reported in China. A study on 10 of those patients found their age at onset of COVID-19 ranged from 112 days to 17 years and clinical presentation was similar to that of adults (24). Some children are afebrile and when fever is present, 
it is typically low. With disease progression, pediatric patients can present with shortness of breath, cyanotic, restless, fatigued, lethargic, and may exhibit poor feeding or show signs of systemic toxicity (24). Children most at risk of COVID-19 appear to be those related to cluster outbreaks or those who had contact with an infected person or recent travel or residence in Wuhan (24).

\subsection{Pregnant Patients}

Pregnant women should be screened, tested, and provided with care in isolation, if required, and adaptations may need to be made to accommodate these persons. The use of any investigational research agents or therapies in pregnant patients requires careful consideration of the safety of mother and fetus and should involve the ethics committee and obstetric specialists (37). Pregnant women with COVID-19 are at risk of hypoxemia that may lead to intrauterine asphyxia and possibly premature delivery (24).

\subsection{Elderly Patients and Patients with Underlying Diseases}

Older patients and those with underlying chronic conditions, such as cardiovascular and respiratory chronic diseases, diabetes, or malignancy, are considered vulnerable. Geriatric patients and/or those with underlying disease are at increased risk for death from acute respiratory distress $(2,9)$. Case-fatality rates among patients in otherwise good health are about $2.3 \%$ (11). Among patients $\geq 80$ years of age, the case-fatality rate is $14.8 \%$, and it is $8.0 \%$ for those between the ages of 70 and 79 years. For critical cases, the case-fatality rate has been reported at $49.0 \%$ (11). Among those with underlying disease (all ages), the case-fatality rates are $10.5 \%$ for cardiovascular diseases, $7.3 \%$ for diabetes mellitus, $6.3 \%$ for chronic respiratory disease, $6.0 \%$ for hypertensives, and 5.6\% for oncology patients (11).

It should be noted that the use of case-fatality rates in describing this epidemic has been criticized because casefatality rates tend to be biased toward the most severe (hospitalized) patients and ignore asymptomatic cases (38). That is, they may suggest more alarming outcomes than are likely. Furthermore, it must be recognized that our data, to date, are based on very small patient populations and may not reflect the future course of the disease (39).

\subsection{Healthcare Professionals}

The COVID-19 appears to follow the pattern of the prior coronavirus epidemics of SARS and MERS such that nosocomial infection is of great concern (21). In mid-February 2020, the Centers for Disease Control and Prevention in China (CDC-China) reported that 3.8\% of all COVID-19 cases were in healthcare workers and, of this population, 63\% resided in Wuhan and $14.8 \%$ of these cases were severe or critical. Five cases with COVID-19 in Wuhan healthcare workers were fatal (11). Healthcare workers have been advised to wear medical overalls, caps, and surgical masks when triaging patients (24). Those working in the pulmonary clinics, infectious disease departments, isolation wards, emergency rooms, or other similar settings should wear medical overalls, caps, disposable isolation clothing, masks, goggles, and a face shield. A respiratory hood may be advised in some situations to prevent splash contamination e.g. when intubating an infected patient is required (24). All protective gear should be done and removed in strict accordance with the hospital's on/off protocols, and protective clothing should not be worn outside of the designated area to prevent cross-contamination (24).

Professionalism and compassion make us remember that healthcare providers at the epicenter of this crisis face tremendous and unprecedented problems ranging from their elevated risk of infection to overwork, frustration, shortage of needed supplies or equipment, unknown aspects of the COVID-19 virus, isolation from family and friends, physical and mental exhaustion, and the stress of dealing with patients in crisis (40). Mental health symptoms such as stress, anxiety, insomnia, depression, anger, and fearfulness not only are detrimental to healthcare professionals, they may also impede their ability to make sound clinical decisions in life-and-death frontline situations (40). Hospitals in Wuhan have initiated special shift scheduling so that clinicians can alternate time in highstress positions and a psychological support team has been set up to provide special interventions, educational efforts, and a hotline for healthcare workers in distress (40).

\section{An Overview of Treatment Strategies}

Overall, the management consists of homeostasis management and the initiation of respiratory support if needed (21). Antimicrobial drugs are only helpful to patients with likely or confirmed bacterial infections and their empirical use is not recommended (21). The role of antiviral medications to treat COVID-19 is not clear, so far (21). Treatment should be supportive care with the patient isolated and monitored for vital signs. It is important that patients stay appropriately hydrated and are well-nourished. Routine blood studies, including C-reactive protein, and organ function should be monitored with other tests as deemed appropriate. Some patients may require oxygen therapy, non-invasive ventilation, or invasive ventilation (41). Extracorporeal membrane oxygenation should be considered in patients with refractory hypoxemia (9). A variety of repurposed drugs, as well as investigational drugs, have been identified for possible use. Screening National Medical Products Administrations approved drug libraries 
and other chemical libraries have been identified as novel agents. Hundreds of clinical trials, including those involving remdesivir, chloroquine, favipiravir, convalescent plasma, and others, are planned or on the way (42).

At present, there is no evidence supporting any drug therapy for the patients (9). The use of antipyretic drugs has been discussed, with some criticism on the use of antiinflammatory medicines that could increase the risk of pulmonary infections. At the moment, there are no data in the literature related to this and, following the current literature, all the antipyretic drugs may be used safely (43). Of course, they should be used with the usual recommendations (44), but especially Ibuprofen seems very safe (45).

There are no antiviral therapies or vaccines for COVID19 (41). In fact, there is no evidence from any randomized clinical studies to support any particular anti-COVID19 therapy (37). Short-term corticosteroids have been used to treat SARS and MERS but current WHO guidelines advise against the use of corticosteroids for COVID-19 unless there are alternate indications for their use (43). Corticosteroids not only suppress pulmonary inflammation but also may suppress the immune response and pathogen clearance (46). Based on a meta-analysis of various treatment modalities for SARS, corticosteroid use in patients with SARS was found harmful (47). In a meta-analysis of 6,548 influenza patients with pneumonia, the risk ratio for mortality increased up to 1.8 (95\% confidence interval, $1.3-2.4, \mathrm{P} \leq$ 0.001 ) among those administered corticosteroids (48). It should be noted that antibiotics are recommended for patients with COVID-19 in case of a bacterial infection (22).

Chloroquine phosphate, a drug that has been used globally for over 70 years to treat malaria, has been shown to have prominent efficacy and safety in studies in China for the treatment of pneumonia associated with COVID19 (49). In in vitro studies, low-micromolar chloroquine concentrations were able to block COVID-19 (49). The halfmaximum effective concentration (EC50) for chloroquine is $1.13 \mu \mathrm{M}$. The half-cytotoxic concentration (CC50) is $>100$ $\mu \mathrm{M}$ (49). In early studies in several Chinese hospitals ( $n=100)$, chloroquine has inhibited the exacerbation of pneumonia, improved lung imaging, promoted a virusnegative conversion, and shortened the course of the disease to a greater extent than control treatment (47). No severe adverse effects were reported (49). Chloroquine has broad-spectrum antiviral activity; for example, it increases the endosomal $\mathrm{pH}$ necessary for virus and cell fusion and likely interferes with the glycosylation of cellular receptors of certain viruses (49-51).

Sepsis has been reported among patients with COVID19. Since septic shock in these patients may be more likely resulted from increased intrathoracic pressure during invasive ventilation, which impedes cardiac filling, this type of septic shock differs from the vasoplegic shock and heart failure that occurs in other patients (46). Although corticosteroids are occasionally used in the treatment of sepsis, it is not clear if they would be effective in treating sepsis related to COVID-19 (46).

\section{Emerging Lessons from COVID-19}

In a retrospective analysis of data on 183 consecutive patients with confirmed cases of the novel coronavirus in the Tongji hospital in China, from the period of January 1 to February 3, 2020, it was found that coagulation parameters may be important predictors of disease trajectory. ${ }^{52}$ Blood samples for coagulation tests were collected on admission and during the hospitalization period, including prothrombin time (PT), activated partial thromboplastin time (APTT), antithrombin activity (AT), fibrinogen, fibrin degradation product (FDP), and D-dimer scores. The mean age in this population was 54.1 years (range 14 to 94) and 41.0\% entered the hospital with a chronic condition such as cardiovascular disease, respiratory disorder, chronic liver or kidney disease, and malignancy. On February 13, 2020, $42.6 \%$ of the patients in this study were discharged, $45.9 \%$ remained hospitalized in stable condition, and $11.5 \%$ ( $\mathrm{n}=$ 21) died (52). The investigators examined anticoagulation parameters between those who survived and the deceased. On admission to the hospital, the non-survivors had significantly lower PT and APTT times and higher D-dimer and FDP levels than did survivors. Toward the end of the hospitalization, fibrinogen and AT levels were significantly lower in non-survivors. This suggests that coagulation parameters may play a predictive role in survival (52). Disseminated intravascular coagulation(DIC) was observed in many deceased patients and DIC may be caused due to sepsis (52).

In other lessons from the COVID-19, it is advisable for public health efforts to educate people about respiratory hygiene. The mouth should be covered when sneezing or coughing and the tissue or paper towel used disposed of immediately, followed by vigorous hand washing (9). People who sneeze and cough often should wear masks to protect those around them (9). Public health messages should also stress the importance (and simplicity) of hand hygiene.

China was able to mobilize rapidly to meet this challenge by isolating the pathogen, creating test protocols and kits, and building dedicated hospitals (53). Developed nations have the resources to meet these challenges, which may be devastating to developing nations with inadequate healthcare resources, lack of funds, and possibly other factors such as ongoing wars or conflicts. For such nations, 
it is crucial to identify cases of the virus rapidly and accurately and to isolate those who might otherwise transmit the disease. This may involve prohibiting travel into or out of the country.

Social media and journalism have been both a blessing and curse in this crisis. Sensationalist reporting, misinformation, politicization of the crisis, and irresponsible messaging may fan the flames of normal watchful concern into an unjustified panic. On the other hand, the rapid sharing of information and accurate reporting have done much to allay fears and allow for a tempered and effective response.

\section{Discussion}

Reports from China suggest that COVID-19 spreads rapidly and the severity of the illness varies among patients from asymptomatic to potentially life-threatening. Moreover, many patients have no fever or abnormal radiological findings (23). Although compared with SARS and MERS, COVID-19 appears to be a milder illness that is more readily transmitted. Since COVID-19 is readily transmitted, higher outbreaks, cases, and fatalities may be expected (18).

The challenges to meeting any sort of epidemic of this nature include tamping the rapid spread of the disease, reducing the high risk of nosocomial infection, tempering the unpredictability of impact, and delivering adequate healthcare resources in a timely fashion (42). In conditions with mild or even moderate symptoms but without proper testing, it can be difficult to differentiate a common cold, influenza, and COVID-19. Nosocomial spread of the disease is challenging because healthcare workers may have extended exposure to the infection (53). This can be associated with a shortage of workers, excessive patient loads, inadequate care for patients, and psychological stress on the clinicians (53).

The nature of the coronavirus and other pathogens in an era of globalization means that healthcare providers, government authorities, and the public must equip themselves to face similar pandemics in the future. The overall goal is to use solid information rather than sensationalism to respond rapidly and effectively address the crisis. Currently, the WHO has issued surveillance definitions and guidelines for the disease that are not discussed here and are likely subjected to updating and modification as COVID-19 is better understood (37).

The COVID-19 is a novel virus with a high potential for rapid spread, especially in our globalized society. However, all of society, especially our "communicators" in the press, media, and healthcare systems, should present scientifically grounded and balanced reporting on the virus rather than sensationalistic stories or histrionic speculation. It is not as if our world is facing a new outbreak of the bubonic plague with no remedies in sight. Rather, we are facing a new virus with a high potential for spread but it has a low potential for being lethal in the normal population. Therefore, announcement, treatment, isolation, and other preventive steps should be taken to stop its spread. These steps are necessary and important, even if they have adverse economic consequences. However, perspective must be maintained and scientific approaches should be considered when describing, discussing, or dealing with COVID19.

\section{Conclusion}

The outbreak of COVID-19 in China at the end of December 2019 has led to a pandemic with numerous infections and some fatalities. This novel coronavirus is spreading rapidly but it is not fatal to most people in otherwise good health who contract the virus. The rapid spread of the virus may be due in part to the fact that it is transmissible even in the latency period and many people have subclinical infections. Fatalities due to COVID-19 appear to be concentrated in vulnerable populations, such as the elderly and those with underlying chronic diseases. Precautions in the general population (rigorous hand hygiene), isolation of those exposed to the disease for a 14-day period, and care in the clinical environment to protect healthcare workers with protective equipment are being used to reduce the spread of COVID-19. In fact, COVID-19 is an evolving epidemic that will be further elucidated as more information and data are collected. Properly addressing this disease will require careful consideration of the scientific facts, effective communication with the public, and strategic planning by governmental entities to ensure that the best possible health outcomes will be realized.

\section{Acknowledgments}

The authors are grateful to L. Di Giacomo for his graphical support and M. Fusco for her contribution to rapidly find scientific material for this paper.

\section{Footnotes}

Authors' Contribution: Jo Ann LeQuang prepared the first draft. All the authors equally contributed to the revision of the manuscript and approved the final version.

Conflict of Interests: None of the authors declared conflict of interest.

Funding/Support: The editing of this paper has been supported by NEMA Res. Group, and Paolo Procacci Foundation with unconditional grants. 


\section{References}

1. World Health Organization. Prioritizing diseases for research and development in emergency contexts. Geneva, Switzerland: World Health Organization,; 2020, [cited March 3]. Available from: https:/www.who.int/activities/prioritizing-diseases-for-researchand-development-in-emergency-contexts.

2. Burki TK. Coronavirus in China. Lancet Respir Med. 2020. doi: 10.1016/s2213-2600(20)30056-4. [PubMed: 32027848].

3. Zhang Y, Xu J, Li H, Cao B. A Novel Coronavirus (COVID-19) Outbreak: A Call for Action. Chest. 2020. doi: 10.1016/j.chest.2020.02.014. [PubMed: 32087216].

4. Ronco C, Navalesi P, Vincent JL. Coronavirus epidemic: preparing for extracorporeal organ support in intensive care. Lancet Respir Med. 2020. doi: 10.1016/s2213-2600(20)30060-6. [PubMed: 32035509].

5. Paules CI, Marston HD, Fauci AS. Coronavirus Infections-More Than Just the Common Cold. Jama. 2020. doi: 10.1001/jama.2020.0757. [PubMed: 31971553].

6. Munster VJ, Koopmans M, van Doremalen N, van Riel D, de Wit E. A Novel Coronavirus Emerging in China - Key Questions for Impact Assessment. N Engl J Med. 2020;382(8):692-4. doi: 10.1056/NEJMp2000929. [PubMed: 31978293].

7. Wang J, Qi H, Bao L, Li F, Shi Y. A contingency plan for the management of the 2019 novel coronavirus outbreak in neonatal intensive care units. Lancet Child Adolesc Health. 2020. doi: 10.1016/s23524642(20)30040-7. [PubMed: 32043976].

8. Zhou P, Yang XL, Wang XG, Hu B, Zhang L, Zhang W, et al. A pneumonia outbreak associated with a new coronavirus of probable bat origin. Nature. 2020. doi: 10.1038/s41586-020-2012-7. [PubMed: 32015507].

9. Jin YH, Cai L, Cheng ZS, Cheng H, Deng T, Fan YP, et al. A rapid advice guideline for the diagnosis and treatment of 2019 novel coronavirus (2019-nCoV) infected pneumonia (standard version). Mil Med Res. 2020;7(1):4. doi: 10.1186/s40779-020-0233-6. [PubMed: 32029004]. [PubMed Central: PMC7003341].

10. Nature. Coronavirus latest: children are as susceptible as adults, study suggests. Nature; 2020, [updated March 5, 2020; cited March 5]. Available from: https://www.nature.com/articles/d41586-020-00154-w.

11. Wu Z, McGoogan JM. Characteristics of and Important Lessons From the Coronavirus Disease 2019 (COVID-19) Outbreak in China: Summary of a Report of 72314 Cases From the Chinese Center for Disease Control and Prevention. Jama. 2020. doi: 10.1001/jama.2020.2648. [PubMed: 32091533].

12. Zoellner D. Coronavirus: Six-week old baby dies of Covid-19, Connecticut governor says. Americas. London, United Kingdom: Independent; 2020, [cited April 13]. Available from: https://www.independent.co. uk/service/contact-us-759589.html.

13. Parveen N. Five-year-old child among latest UK coronavirus deaths. Coronavirus outbreak. London, United Kingdom: The Guardian; 2020, [cited April 13]. Available from: https://www.theguardian.com/uknews/2020/apr/04/five-year-old-child-among-latest-ukcoronavirus-deaths.

14. Woodward A. Coronavirus: One-day-old baby dies of Covid-19 complications in Louisiana, coroner says. Americas. London, United Kingdom: Independent; 2020, [cited April 13]. Available from: https://www. independent.co.uk/news/world/americas/coronavirus-baby-deathlouisiana-children-premature-pregnancy-a9451261.html.

15. Gagneur A, Vallet S, Talbot PJ, Legrand-Quillien M, Picard B, Payan C et al. Outbreaks of human coronavirus in a paediatric and neonatal intensive care unit. European Journal of Pediatrics. 2008;167(12):142734. doi: 10.1007/s00431-008-0687-0.

16. Channappanavar R, Perlman S. Pathogenic human coronavirus infections: causes and consequences of cytokine storm and immunopathology. Semin Immunopathol. 2017;39(5):529-39. doi: 10.1007/s00281-017-0629-x. [PubMed: 28466096].

17. Huang C, Wang Y, Li X, Ren L, Zhao J, Hu Y, et al. Clinical features of patients infected with 2019 novel coronavirus in Wuhan, China.
Lancet. 2020;395(10223):497-506. doi: 10.1016/s0140-6736(20)30183-5. [PubMed: 31986264].

18. Li G, Fan Y, Lai Y, Han T, Li Z, Zhou P, et al. Coronavirus infections and immune responses. J Med Virol. 2020;92(4):424-32. doi: 10.1002/jmv.25685. [PubMed: 31981224].

19. Zhu N, Zhang D, Wang W, Li X, Yang B, Song J, et al. A Novel Coronavirus from Patients with Pneumonia in China, 2019. $N$ Engl J Med. 2020;382(8):727-33. doi:10.1056/NEJMoa2001017. [PubMed: 31978945].

20. Lu R, Zhao X, Li J, Niu P, Yang B, Wu H, et al. Genomic characterisation and epidemiology of 2019 novel coronavirus: implications for virus origins and receptor binding. Lancet. 2020;395(10224):565-74. doi: 10.1016/s0140-6736(20)30251-8. [PubMed: 32007145].

21. Wang C, Horby PW, Hayden FG, Gao GF. A novel coronavirus outbreak of global health concern. Lancet. 2020;395(10223):470-3. doi: 10.1016/S0140-6736(20)30185-9. [PubMed: 31986257]. [PubMed Central: PMC7135038].

22. Cheng ZJ, Shan J. 2019 Novel coronavirus: where we are and what we know. Infection. 2020;48(2):155-63. doi: 10.1007/s15010-020-01401-y. [PubMed: 32072569].

23. Guan W, Ni Z, Hu Y, Liang W, Ou C, He J, et al. Clinical Characteristics of Coronavirus Disease 2019 in China. New England Journal of Medicine. 2020. doi: 10.1056/NEJMoa2002032.

24. Chen ZM, Fu JF, Shu Q, Chen YH, Hua CZ, Li FB, et al. Diagnosis and treatment recommendations for pediatric respiratory infection caused by the 2019 novel coronavirus. World J Pediatr. 2020. doi: 10.1007/s12519-020-00345-5. [PubMed:32026148].

25. Viboud C, Lessler J. The 1918 Influenza Pandemic: Looking Back, Looking Forward. Am J Epidemiol. 2018;187(12):2493-7. doi: 10.1093/aje/kwy207. [PubMed: 30346477]. [PubMed Central: PMC6454441].

26. Peng PW, Ho P, Hota SS. Outbreak of a new coronavirus: what anaesthetists should know. British Journal of Anaesthesia. 2020. doi: 10.1016/j.bja.2020.02.008.

27. Jiang S, Shi Z. The First Disease $X$ is Caused by a Highly Transmissible Acute Respiratory Syndrome Coronavirus. Virologica Sinica. 2020. doi: 10.1007/s12250-020-00206-5.

28. The Lancet. COVID-19: fighting panic with information. Lancet. 2020;395(10224):537. doi: 10.1016/s0140-6736(20)30379-2. [PubMed: 32087777].

29. Noack R, Berger MJ, Horton A, Freedman A. Live updates: Cruise ship held off California coast; coronavirus turmoil grows as U.S. death toll mounts. The Washington Post. Washington, D.C: The Washington Post; 2020, [updated March 5, 2020]. Available from: https://www. washingtonpost.com/world/2020/03/05/coronavirus-live-updates/.

30. Taylor A. Trump signs 8.3B USD bill to combat coronavirus outbreak in US. Associated Press; 2020, [cited March 7, 2020]. Available from: https: //apnews.com/30fc0af2ffb9320e1d8fa6bb6d8b23a1.

31. World Health Organizaiton. Coronavirus disease 2019 (COVID-19) Situation Report-44. World Health Organization; 2020, [cited March 5, 2020]. Available from: https://www.who.int/docs/defaultsource/coronaviruse/situation-reports/20200304-sitrep-44-covid19.pdf?sfvrsn=783b4c9d_6.

32. Xu H, Rebaza A, Sharma L, Dela Cruz CS; Chang. Protecting healthcare workers from subclinical coronavirus infection. Lancet Respir Med. 2020;8(3). e13. doi: 10.1016/S2213-2600(20)30066-7. [PubMed: 32061333]. [PubMed Central: PMC7128440].

33. Wu JT, Leung K, Leung GM. Nowcasting and forecasting the potential domestic and international spread of the 2019-nCoV outbreak originating in Wuhan, China: a modelling study. Lancet. 2020;395(10225):689-97. doi: 10.1016/s0140-6736(20)30260-9. [PubMed: 32014114].

34. Wax RS, Christian MD. Practical recommendations for critical care and anesthesiology teams caring for novel coronavirus (2019nCoV) patients. Can J Anaesth. 2020. doi: 10.1007/s12630-020-01591-x. [PubMed: 32052373]. 
35. Wu J, Wu X, Zeng W, Guo D, Fang Z, Chen L, et al. Chest CT Findings in Patients with Corona Virus Disease 2019 and its Relationship with Clinical Features. Invest Radiol. 2020. doi: 10.1097/rli.0000000000000670. [PubMed: 32091414].

36. Chung M, Bernheim A, Mei X, Zhang N, Huang M, Zeng X, et al. CT Imaging Features of 2019 Novel Coronavirus (2019-nCoV). Radiology. 2020:200230. doi: 10.1148/radiol.2020200230. [PubMed: 32017661].

37. World Health Organization. Clincial management of severe acute respiratory infection when novel coronavirus (2019-nCoV) infection is suspected: Interim Guidance. Geneva, Switzerland: World Health Organization; 2020, [cited March 5]. Available from: https://www.who.int/publications-detail/clinical-managementof-severe-acute-respiratory-infection-when-novel-coronavirus(ncov)-infection-is-suspected.

38. Xu Z, Li S, Tian S, Li H, Kong LQ. Full spectrum of COVID-19 severity still being depicted. Lancet. 2020. doi: 10.1016/s0140-6736(20)30308-1. [PubMed:32066525].

39. Gu X, Cao B, Wang J. Full spectrum of COVID-19 severity still being depicted - Authors' reply. Lancet. 2020. doi:10.1016/s0140-6736(20)303718. [PubMed: 32066526].

40. Kang L, Li Y, Hu S, Chen M, Yang C, Yang BX, et al. The mental health of medical workers in Wuhan, China dealing with the 2019 novel coronavirus. Lancet Psychiatry. 2020;7(3). e14. doi: 10.1016/S2215-0366(20)30047-X. [PubMed: 32035030]. [PubMed Central: PMC7129673].

41. Arabi YM, Fowler R, Hayden FG. Critical care management of adults with community-acquired severe respiratory viral infection. Intensive Care Med. 2020;46(2):315-28. doi: 10.1007/s00134-020-05943-5. [PubMed: 32040667]. [PubMed Central: PMC7079862].

42. World Health Organization. Report of the WHO-China Joint Mission on Coronavirus Disease 2019 (COVID-19). World Health Organization; 2020, [cited March 9, 2020]. Available from: https://www.who.int/docs/default-source/coronaviruse/whochina-joint-mission-on-covid-19-final-report.pdf.

43. Varrassi G. Warning Against the Use of Anti-Inflammatory Medicines to Cure COVID-19: Building Castles in the Air. Adv Ther. 2020. doi: 10.1007/s12325-020-01321-1. [PubMed: 32239459]. [PubMed Central: PMC7110984].

44. Varrassi G, Alon E, Bagnasco M, Lanata L, Mayoral-Rojals V, Paladini A, et al. Towards an Effective and Safe Treatment of Inflammatory
Pain: A Delphi-Guided Expert Consensus. Adv Ther. 2019;36(10):261837. doi:10.1007/s12325-019-01053-x. [PubMed: 31485978]. [PubMed Central: PMC6822819].

45. Varrassi G, Pergolizzi JV, Dowling P, Paladini A. Ibuprofen Safety at the Golden Anniversary: Are all NSAIDs the Same? A Narrative Review. Adv Ther. 2020;37(1):61-82. doi: 10.1007/s12325-019-01144-9. [PubMed: 31705437].

46. Russell CD, Millar JE, Baillie JK. Clinical evidence does not support corticosteroid treatment for 2019-nCoV lung injury. Lancet. 2020;395(10223):P473-5.

47. Stockman LJ, Bellamy R, Garner P. SARS: systematic review of treatment effects. PLoS Med. 2006;3(9). e343. doi: 10.1371/journal.pmed.0030343. [PubMed: 16968120]. [PubMed Central: PMC1564166].

48. Ni YN, Chen G, Sun J, Liang BM, Liang ZA. The effect of corticosteroids on mortality of patients with influenza pneumonia: a systematic review and meta-analysis. Crit Care. 2019;23(1):99. doi: 10.1186/s13054019-2395-8. [PubMed: 30917856]. [PubMed Central: PMC6437920].

49. Gao J, Tian Z, Yang X. Breakthrough: Chloroquine phosphate has shown apparent efficacy in treatment of COVID-19 associated pneumonia in clinical studies. Biosci Trends. 2020. doi: 10.5582/bst.2020.01047. [PubMed: 32074550].

50. Savarino A, Boelaert JR, Cassone A, Majori G, Cauda R. Effects of chloroquine on viral infections: an old drug against today's diseases? Lancet Infect Dis. 2003;3(11):722-7. doi: 10.1016/s1473-3099(03)00806-5. [PubMed: 14592603].

51. Yan Y, Zou Z, Sun Y, Li X, Xu KF, Wei Y, et al. Anti-malaria drug chloroquine is highly effective in treating avian influenza A H5N virus infection in an animal model. Cell Res. 2013;23(2):300-2. doi: 10.1038/cr.2012.165. [PubMed: 23208422]. [PubMed Central PMC3567830].

52. Tang N, Li D, Wang X, Sun Z. Abnormal coagulation parameters are associated with poor prognosis in patients with novel coronavirus pneumonia. J Thromb Haemost. 2020;18(4):844-7. doi: 10.1111/jth.14768. [PubMed: 32073213]. [PubMed Central: PMC7166509].

53. Liu Y, Li J, Feng Y. Critical care response to a hospital outbreak of the 2019-nCoV infection in Shenzhen, China. Crit Care. 2020;24(1):56 doi: 10.1186/s13054-020-2786-x. [PubMed: 32070391]. [PubMed Central: PMC7029610]. 